\title{
Precise Assessment of Self-Selected Gait Velocity in Domestic Environments
}

\author{
Thomas Frenken*, Mehmet Gövercin ${ }^{\dagger}$, Sebastian Mersmann ${ }^{\dagger}$, Prof. Dr.-Ing. Andreas Hein ${ }^{\ddagger}$ \\ *OFFIS - Institute for Information Technology, Escherweg 2, D-26121 Oldenburg, Germany, Email: thomas.frenken@offis.de \\ †Geriatrics Research Group, Charité - Universitätsmedizin Berlin, D-13347 Berlin, Germany

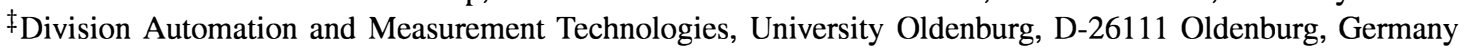

\begin{abstract}
A novel approach to precise and reliable computation of self-selected gait velocity in domestic environments based on the measurements of a laser range scanner is presented. The computation does not require a priori knowledge of the environment. The sensor is part of an ambient assessment system under development which is meant to objectively measure and compare capacity and performance in mobility. Possible application fields may be early detection or differential diagnosis in dementia and assessment of fall risk. Regarding the challenges of future health systems due to the demographic change delaying need of care or preventing falls in dementia can help decrease costs while increasing perceived quality of life for people concerned and their carers. Within this paper we present our theoretic concept and algorithm for computing self-selected gait velocity utilizing measurement from a laser range scanner. Results of an experiment show that the laser range scanner delivers precise measurement and in the future may be used for absolutely unobtrusive analysis of spatio-temporal parameters of gait even in demented people.
\end{abstract}

Index Terms-mobility assessment, gait velocity assessment, laser range scanner, LIDAR, smart homes, ambient sensors

\section{INTRODUCTION}

A person's mobility is closely connected to his or her perceived quality of life and a fundamental requirement for an independent lifestyle [1]. Starting at the age of 60 years, elderly people expose a slower gait velocity [2]. This agerelated change in mobility is not pathological. Nevertheless, many pathologic diagnoses can be directly deduced from an impaired mobility [3]. The most obvious impairment visible even to layman is a reduced self-selected gait velocity.

Due to the demographic change especially industrial countries are steering towards a graying society. In 2050 approximately $16.4 \%$ of the wordwide population and $27.6 \%$ of the European population will be older than 65 years, while in the year 2000 it have been $6.9 \%$ respecitelvy $14.7 \%$ [4]. This change poses many problems due to the decline of the care ratio (ratio of people aged 20-65 years to those aged older than 65 years). Regarding Germany, in $200561 \%$ of the population has been in working age and only $19 \%$ was in retirement age. For 2050 these numbers are estimated to change to $50 \%$ respectively $30 \%$ [5]. Considering that elderly people have a proportionally higher demand on health services, this ratio change leads to less people paying taxes for financing the health care system while there will be more people requiring health services. Costs due to need of care of demented people and by their high fall risk are two of the major factors influencing the proportionally higher costs to the health care system caused by elderly people. Dementia is an old-age disease. Starting at the age of 60 years the prevalence reduplicated every five years reaching $13 \%$ for octogenarians and up to $45 \%$ for those aged 95 years and older [6]. Due to the demographic change, the total amount of demented persons will increase. In the year 2010, approximately 35.6 million people worldwide will suffer from dementia. The amount will nearly reduplicate each 20 years, reaching 115.4 million people in 2050 [7]. Falls are a major problem in demented people. $30 \%$ of all European elderly aged $65+$ years and $50 \%$ of those older than 80 years fall once a year [8]. Demented elderly have a proportionally higher risk of falling [9]. Overall direct and indirect costs imposed to the health system due to the need of care by demented persons were 315.4 billion US-Dollar in 2005 [10]. In order to meet the increased challenges on the health systems imposed by the demographic change and the overall increasing number of demented people, new approaches for delaying the need of care and for prevention of acute incidents like falls need to be developed. Long-term monitoring of mobility may provide the required means. Mobility impairments have a high prevalence in dementia [11] and are an early indicator [12]. Severity of gait and balance disorders increases with severity of neurological disorders [13]. Research suggests that certain gait disorders prevail in certain types of dementia [11] and might thus be used for differential diagnosis. Gait and balance disorders have shown being related to a higher risk of falling. Especially slow self-selected gait velocity has found being related to an increased risk for falls, admission to hospital, and need of care [14].

Nevertheless, the full potential of long-term mobility monitoring is currently not exploited. Nowadays mobility is often only assessed after an acute incidence took place, most often by utilizing geriatric assessments which require personal supervision by an expert. This is mainly because domestic environments are only fractionally integrated into health care systems. It is widely expected that only through use of technology more efficient health care can be realized [15]. Therefore, various approaches to mobility telemonitoring have been developed utilizing either wearable sensors or ambient sensors. Nevertheless, existing approaches have serious limitations especially regarding the monitoring of demented people. Additionally, 
most systems do only assess capacity or performance in mobility. Comparison of objective measurements regarding both is required in order to reliably detect environmental factors and pathological causes.

Within this paper we present our first work towards a mobility assessment system for objective measurement of capacity and performance in mobility. The system is exclusively based on ambient sensors and requires no interaction with the patients monitored. Light barriers are used for assessment of general trends in mobility and a laser range scanner is utilized for detailed gait analysis. We establish a novel concept of assessing gait velocity using measurement of a laser range scanner. An experiment was conducted within a domestic environment in order to compare usage of light barriers and laser range scanner for gait velocity assessment comparing precision of measurements and opportunities for detailed gait analysis.

\section{Medical Motivation}

The International Classification of Functioning, Disability and Health (ICF) from the World Health Organization (WHO) lists mobility as one of nine domains in the component "Activities and Participation". Mobility has two aspects dealing directly with the human. These are "changing and maintaining body position (d410-d429)" and "walking and moving (d450-d469)". Two qualifiers describe resources in mobility: performance and capacity. Performance describes what an individual does in his or her current environment regarding all natural environmental influences. Capacity describes what an individual is able to do under ideal circumstances and may thus only be measured in a standardized environment excluding as many environmental factors as possible. An identified gap between capacity and performance may guide the conclusion on whether reduced performance is caused by environmental influences or pathological reasons.

Human gait patterns change with increased age. Starting at the age of 60-70 years, self-selected gait velocity decreases each decade by $12 \%-16 \%$ during self-imposed activities. The decrease is often caused by a reduced step length whereas the step frequency remains stable. This age-related change in gait patterns is meant to contribute to a more stable gait, it is not pathological [2]. A recent clinical study with more than 700 particpants aged between 20 and $90+$ years has found an average gait velocity during a six metre walk of $1.1 \mathrm{~m} / \mathrm{s}$ for men aged between $75-89$ years, decreasing to $0.9 \mathrm{~m} / \mathrm{s}$ when being even older. Women aged 75-79 years were found to walk with an average velocity of $1.1 \mathrm{~m} / \mathrm{s}$, respecitvely $1.0 \mathrm{~m} / \mathrm{s}$ when aged $80-84$ years and $0.8 \mathrm{~m} / \mathrm{s}$ when being older than 85 years [16]. Impairments of mobility due to pathological reasons lead to more significant changes in parameters of gait than age-related changes [2]. Therefore, significant long-time changes in mobility may point to pathological causes and may thus be utilized for early diagnosis.

One of the most frequent pathological reasons for mobility impairments are neurological diseases, especially dementia [13]. Prevalence of gait disorders for 245 neurological patients was $93 \%$ when being diagnosed with Parkison's disease with dementia, 79\% with vascular dementia, $75 \%$ with dementia with Lewy Bodies, $43 \%$ with Parkinson's disease without dementia, and 25\% with Alzheimer's disease [11]. In general, severity of gait and balance disorders increased with severity of neurological disorders [13]. Comparing studies showed that Alzheimer patients had the least severe disorders, patients with Parkinson's disease without dementia were slightly more effected. All other patient groups had more severe disorders but using the Tinetti mobility assessment no significant differences among the remaining groups were found [11]. Step-to-step variability in gait parameters of demented people seems to be more specific and sensitive than changes in mean values of gait parameters [17].

In certain dementia types and stages different higher-level gait disorders may prevail. Frontal gait disorders are specific with $87 \%$ for vascular dementia with a sensitivity of $76 \%$. Parkinsonian gait points to dementia with Lewy Bodies or Parkinson's disease with a specifity of $84 \%$ and a sensitivity of $87 \%$ [11].

Due to their gait and balance disorders, dementia patients show an increased risk for falls [9]. In clinical studies, self-selected gait velocity slower than $0.7 \mathrm{~m} / \mathrm{s}$ was related to an increased risk for falls, admission to hospital, and need of care [14].

From a clinical perspective long-term monitoring of changes in mobility has a high-potential for early diagnosis of various diseases and for assessment of fall risk [17]. In todays health systems this potential is most often not exploited, especially regarding demented people. Rather, mobility assessments are only applied infrequently or after an acute incident like a fall took place. In order to enable prevention of those incidents or delay need of care mobility assessment needs to be technically supported in professional as well as in domestic environments. Comparing performance in professional environments and capacity in the domestic environment may provide new insights. On a more personal level early detection may help furthering an independent lifestyle by enabling early and purposeful prevention and may therefore increase quality of life for affected people, relatives, and carers.

\section{State of the ART}

Today, mobility of elderly people is often assessed using geriatric assessments. A geriatric assessment is a multidimensional process designed to assess an elderly person's functional ability, physical health, cognitive and mental health, and socio-environmental situation [18]. The most frequently used assessment from the field of mobility is probably the TimedUp \& Go [19]. In case of severe injuries or problems with prostheses or implants laboratories equipped with camerabased systems for cinematic gait analysis based on marker tracking, fluoroscopy systems, systems for cinetic gait analysis of ground reaction forces utilizing force platforms, and dynamic electromyography may be utilized. Recent research investigated mobility telemonitoring directly in the home of affected people. Two main approaches arose using either wearable sensors or sensors installed into the environment [20]. For the state of the art we explicitly focus on research assessing at 
least one spatio-temporal parameter of gait, therefore leaving out systems measuring general activity. A special focus is on monitoring elderly or demented people.

\section{A. Wearable Assessment Technologies}

Wearable sensors may be placed either on one or many positions directly on the body or in cloth and objects worn. Several wearable sensors are also referred to as body area networks (BAN). The most frequently used wearable sensors are accelerometers and gyroscopes. In recent research there is a clear trend towards combining several sensors into one sensor node placed on the person's center of mass instead of placing single sensors on various positions of the body.

Accelerometers and gyroscopes have been applied to gait phase detection and measurement of various parameters of gait. Stance and swing of the human gait cycle have been detected with an overall accuracy of up to $98 \%$ utilizing three uniaxial accelerometers underneath the knee [21]. Detection of five gait phases worked with an overall accurcy of up to 86-91\%. Menz et al. [22] measured various parameters of gait, e.g. walking velocity, cadence, average step length, and step timing variability, while walking on irregular surface. Two triaxial accelerometers were placed on a helmet and on a belt near the sacrum. They concluded that older adults adopted a more conservative gait pattern resulting in reduced gait velocity and step length in order to maintaine balance despite age-related deficity in physiological function. Auvinet et al. [23] utilized two accelerometers and a recording device placed on a belt in the middle of the lower back of 282 healthy adults aged betwen 20 and 98 years. Various parameters, including gait velocity and stride length, were found to be age-dependent and being higher in men than in women starting to decrease in the sixth respectively in the seventh decade. Similar results were obtained by Zijlstra [24] and colleagues placing a triaxial accelerometer on the dorsal side of the trunk. They found a limited range of walking, a smaller step length, and a higher variability of temporal parameters in older participants.

Pressure sensors under or integrated into the sole of shoes, later combined with accelerometers and gyroscopes, have been used to measure pressure distribution on certain points of the feet in order to infer gait phases or detect abnormal gait patterns. Zhu et al. [25] developed a wired shoe insole with seven pressure sensors each in order to determine the role of pressure in causing damage to insensate diabetic feet and to distinguish between normal and shuffling gait [26]. Hausdorff et al. [27] placed only two pressure sensors in each insole and were able to detect start and end of gait cycles with an accuracy of up to $97 \%$ compared to measurement of a force platform. Skelly et al. [28] used four pressure sensors, Pappas et al. [29] three pressure sensors in each insole in order to detect five respectively four gait phases and compute the strength of the required stimuli for functional electrical stimulation (FES) systems. Hanlon and Anderson [30] combined a footswitch and two accelerometers placed on a foot and knee in order to further enhance algorithms for gait event detection, especially initial contact (IC), for FES stimuli computation. They found that footswitch systems must be recommended over accelerometer systems for accurate detection of IC, at the moment. Bamberg et al. [31] placed overall 14 sensors in and on a shoe in oder to detect heel-strike and toe-off during gait. Huang et al. [32] used nine sensors in order to detect five gait phases with an accuracy of up to $91 \%$. The shoe was later on used to detect four abnormal gait disorders [33]. Recently Kong et al. published a series of papers on a system incorporating only four pressure sensors in each insole in order to reliably detect all eight gait phases which may be used to infer several gait disorders [34].

\section{B. Ambient Assessment Technologies}

Ambient sensors are integrated into the environment or in objects used by the person monitored. Environments equipped with such sensors are also referred to as health smart homes [20]. Very few systems for detailed mobility analysis based on ambient sensors have been described so far. This might be due to the difficulty of measuring relevant parameters using available ambient sensors.

Chan et al. [35] used active motion sensors and magnetic contacts placed in door frames in order to determine a person's walking direction, while passive motion sensors placed on the ceiling were used for localization purposes. The gathered information was used to determine a person's walking distance per day. Optical and ultrasonic sensors placed in door frames to determine a person's walking speed and direction each time he or she passed were used by Cameron et al. [36]. The application field was estimation of fall risk. Pavel et al. [37] published a series of papers on a system based on passive motion sensors placed in various rooms of a flat. First, gait velocity was computed dividing known distance between rooms by measured transition times. In order to generate more reliable results, they additionally placed three passive motion sensors into a frequently used area within the flat. Transition times between the sensors were recorded and used to compute more reliable gait velocity values. However, a sufficient long area with a restricted motion path has to be available in a flat in order employ this approach.

Recent technical advances have enabled researchers to build large sensor mats. Applications include analysis of dance steps [38] and identification of persons based on analysis of various parameters of gait like step width and cadence utilizing a sensor mat with 1536 binary sensors [39].

Tung et al. [40] have equipped a walker with several sensors in order to assess mobility of a person. The iWalker is equipped with optical sensors for measuring wheel rotation and moving direction, a six-dimensional gyroscope and accelerometer measuring speed and distance, several load cells in the handles and on the frame measuring weight distribution and propulsion forces, and a portable camera for recording the environment while walking. 


\section{Comparison of Technologies}

With regard to our objective of assessing capacity and performance in mobility of demented people the different approaches to mobility monitoring have advantages and disadvantages regarding reliability and precision of measurements and applicability in different environments.

Clinical assessments have been widely adopted in geriatrics. Nevertheless, they only provide a short-term inside into the capacity of a person and are often perceived as some kind of test resulting in patients performing at their best. Despite standardized descriptions professionals perform assessments differently. There is only very little technical support for clinical assessments, therefore manual supervision and documentation of the results are required. Clinical assessments do only detect existing impairments, they do most often not provide a detailed insight into the reasons for these impairments.

Technical advance has led to wearable sensors with reduced size, weight, and costs. Wearables can provide detailed biomechanical information about the person wearing the device, ideally in any environment. Nevertheless, most wearable sensors are not suitable for unsupervised use by layman or demented people. Wearables require direct interaction, therefore donning, charging, or operating the device every day. Not or incorrectly donning the device heavily influences the measurements. Studies indicate that simply being aware of wearing a sensor may influence the measurement results [41]. Mobility monitoring utilizing ambient sensors has been rather imprecise so far. Nevertheless, ambient sensors are totally unobtrusive and suited even for demented persons. Ideally, monitored persons do not recognize present sensors in their everyday life, thus measurements might be more reliable. Ambient sensors may monitor several persons in their coverage at the same time. However, identifying the monitored persons is often difficult. This issue has been addressed by using RFID technology [37], height recognition [35], footstep analysis [42], and audio recognition [43]. Installation of sensors may be costly. Combination systems of wearable and ambient sensors combine advantages and disadvantages of both approaches.

Laboratory equipment for mobility monitoring provides the most precise measurements, so far. Nevertheless, the equipment is too large or complicated for being applied outside of a large laboratory and can only be handled by experts. Some systems require the patient to perform difficult calibration tasks which are not suitable for cognitive impaired or elderly people.

In conclusion, a system based on ambient sensors is desirable due to its unobtrusiveness and suitability for monitoring even demented people but current solutions do not provide precise measurements which are sufficient for detailed diagnoses of e.g. gait disorders. Additionally, deployment in environments with more than one person is difficult due to identification purposes. Existing systems require a priori knowledge about the environment e.g. distances between rooms in order to compute desired output variables.

\section{APPROACH}

Our novel approach to mobility monitoring is based on the comparison of capacity and performance regarding mobility measured by a combination of ambient sensors. Light barriers for measurement of general trends in mobility mainly in the home environment and a very precise ambient sensor, a laser range scanner. We hypothesize that laser range scanners are applicable for precise measurement of capacity regarding mobility in an environment mainly free of environmental influences like a hospital as well as for measuring performance in a domestic environment. Gathered information should be sufficient for analyzing various spatio-temporal parameters of gait and even for diagnosis of various gait disorders. Additionally, there is no need for the patients to interact with the sensor which may lead to more reliable results and makes the sensor even suitable for measuring mobility of cognitively impaired people. As a first step towards realizing the desired assessment system, within this paper we focus on our approach to measuring self-selected gait velocity. The approach does not require a priori knowledge about the environment and delivers reliable and precise results.

Figure 1 shows the principle of computing a person's movement trajectory from measurements taken by a laser range scanner at the height of the subject's legs. Briefly said, the gait velocity $v_{k}$ for point in time $T_{0} * k$ is computed by dividing the distance walked $d_{k}$ by the time elapsed $T_{0}$. The distance $d_{k}$ is the length of the vector pointing to the difference in range means between two consecutive measurement sets. Therefore, computing the self-selected gait velocity does not necessarily include differentiating the two legs of a subject. However, depending on the position of the laser range scanner relative to the subject measured, trajectory and gait velocity computed based on the measurements may differ from the trajectory defined by the subject's center of mass and the actual gait velocity due to two reasons. First, the laser range scanner measures only the surface of legs facing the scanner. The scanner is not capable of measuring the depth of an object. Therefore, the computed mean distance vector will point to a position slightly more into the direction of the laser range scanner than the vector pointing to the real center of mass. The difference in these two vectors depends on the depth of the object measured. Second, looking from the position of the laser range scanner one leg may be covered by the other while walking, especially during the gait phases mid stance and initial swing of the human gait cycle [44]. The computed mean distance for such measurements differs from those including both legs resulting in either a longer or shorter mean distance depending on the leg covered. However, being aware of those limitations an approximated gait velocity may be computed in a process involving three steps: environment recognition, dynamic object measurement, and gait velocity computation.

\section{A. Environment Recognition}

Prerequisite for measuring the movement trajectory of a subject is the ability to distinguish moving objects like humans 


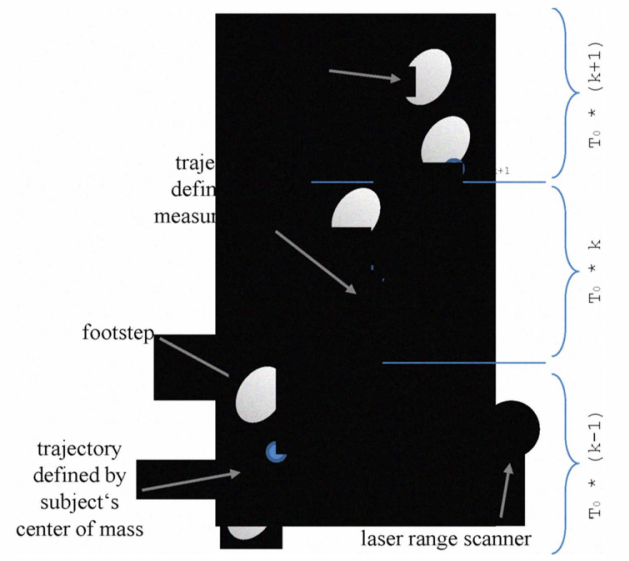

Fig. 1. Principle of computing gait velocity from laser range scanner's measurements

from stationary objects. This may be achieved by measuring ranges within the static environment before any dynamic object enters. In order to compensate for noise, caused by the scanner's hardware itself, our approach is to compute the mean range $\bar{r}^{c}(\alpha)$ and the standard deviation $\sigma^{c}(\alpha)$ from measurement values $r_{k, \alpha}^{c}$. Values $r_{k, \alpha}^{c}$ define the range measured for each angle $\alpha$ in the measurement sector $\left[\operatorname{start}_{\alpha}, e n d_{\alpha}\right]$ over a given number $k^{c}$ of measurement sets by the scanning function $\operatorname{scan}(k, \alpha)$. The number of required measurement sets $k^{c}$ for the calibration may vary among scanner models. According to a Gaussian distribution, $68.27 \%$ of all measured values for the corresponding measurement angle $\alpha$ fall into the interval $\left[\bar{r}^{c}(\alpha)-\sigma^{c}(\alpha), \bar{r}^{c}(\alpha)+\sigma^{c}(\alpha)\right]$. Therefore, computed mean range $\bar{r}^{c}(\alpha)$ and standard deviation $\sigma^{c}(\alpha)$ can be used to distinguish static from dynamic objects during measurements and for filtering most noise.

\section{B. Dynamic Object Measurement}

During a measurement process computed values $\bar{r}^{c}(\alpha)$ and $\sigma^{c}(\alpha)$ may be used for differentiating static and dynamic objects. Range values $r_{k, \alpha}$ measured by the scanning function $\operatorname{scan}(k, \alpha)$ lying in between the defined interval $\left[\bar{r}^{c}(\alpha)-\sigma^{c}(\alpha), \bar{r}^{c}(\alpha)+\sigma^{c}(\alpha)\right]$ may be treated as static objects. The value of $r_{k, \alpha}$ will be NaN (Not a Number) for those measurements falling into the interval, therefore excluding them from the following computations, or the measured range otherwise. For visualization purposes measured values are also transformed into a cartesian coordinate system during measurement resulting in a position vector $\vec{p}_{k, \alpha}$ pointing to the location of a detected moving object for each angle $\alpha$ and measurement set $k$. Depending on the preferred orientation of the coordinate system, $\alpha$ may be rotated prior to the transformation. Formula 1 shows computation of the position vector $\vec{p}_{k, \alpha}$ dependent on the value of the range $r_{k, \alpha}$ measured by the scanning function $\operatorname{scan}(k, \alpha)$.

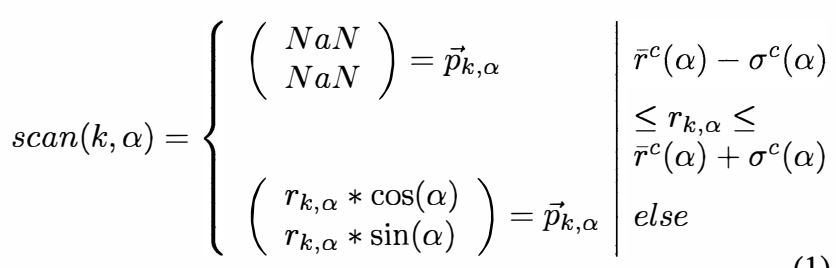

\section{Gait Velocity Computation}

Using the measured position vector $\vec{p}_{k, \alpha}$ for each measurement angle $\alpha$ and measurement set $k$ the movement trajectory and the self-selected gait velocity of the subject measured may be approximated. For each measurement set $k$ the mean range vector $\vec{r}_{k}$ computed points approximately to the subject's center of mass at the point in time $T_{0} * k$. The distance walked $d_{k}$ between two measurement sets $k$ and $k-1$ is approximately the length of the vector $\vec{d}_{k}$ between two consecutive mean range vectors $\vec{r}_{k}$ and $\vec{r}_{k-1}$. Dividing $d_{k}$ by the time elapsed $T_{0} * k-T_{0} *(k-1)$ between the two corresponding measurement sets $k$ and $k-1$ gives approximately the self-selected gait velocity $v_{k}$ for point in time $T_{0} * k$. Formula 2 sums up the previous steps. Applying an additional mean filter to all computed velocity values $v_{k}$ within one second gives the approximated gait velocity per second.

$$
v_{k}=\frac{\left|\left(\frac{\sum_{\alpha=\text { start }}^{e n d_{\alpha}} \vec{p}_{k, \alpha}}{\mid \text { end } d_{\alpha}-\text { start }_{\alpha} \mid}\right)-\left(\frac{\sum_{\alpha=\text { start }}^{\text {end }} \vec{p}_{k-1, \alpha}}{\mid e n d_{\alpha}-\text { start }_{\alpha} \mid}\right)\right|}{T_{0}}
$$

An experiment for comparing feasibility and precision of measuring self-selected gait velocity using light barriers and a laser range scanner was conducted. The experiment took place in a living lab in Oldenburg, Germany. The floor plan of the living lab is shown in figure 3. A Hokuyo URG-04LX-UG01 laser range scanner and two IR light barriers were used for the experiment. The URG-04LX-UG01 includes a safety class 1 semiconductor laser diode with a wavelength of $785 \mathrm{~nm}$ that measures ranges from $20 \mathrm{~mm}$ to approximately $4000 \mathrm{~mm}$ with an accuracy of $30 \mathrm{~mm}$ to $3 \%$ of measurement above $1000 \mathrm{~mm}$ range. Each series of measurements takes 100 milliseconds and covers a scan angle of 240 degrees with an angular resolution of 0.36 degrees. However, for the experiment measurements were taken with an angular resolution of only 1 degree. Two common IR light barriers were combined with a FS20 tramitter in order to wirelessly transmit "on" and "off" states to an FHZ1000PC FS20 base station. The FHZ1000PC and the Hokuyo URG-04LX-UG01 were connected to a PC using the USB port.

\section{A. Methods}

Five healthy people aged 25-39 participated in the experiment. For each participant ten measurement sets were recorded while walking along two paths within the flat's entrance hall. The laser range scanner was placed at a height of $38 \mathrm{~cm}$ in 


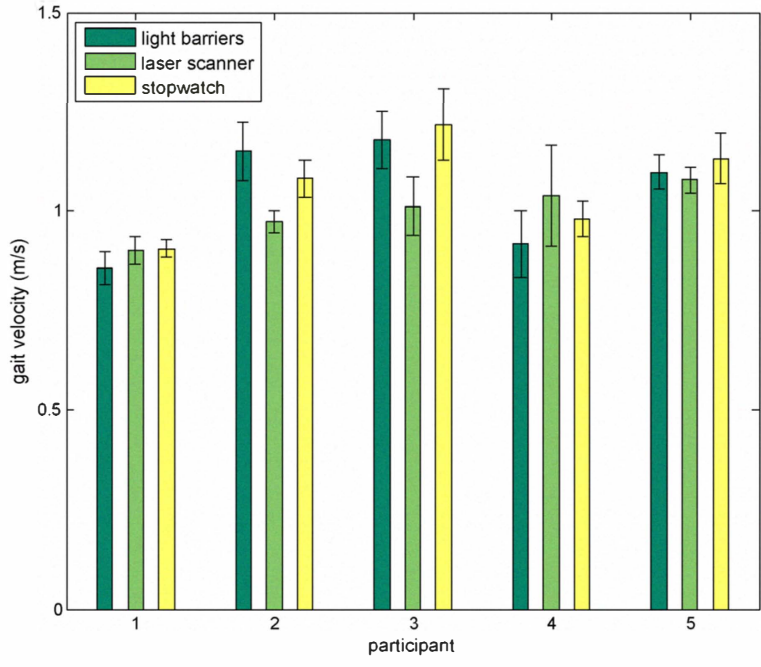

Fig. 2. Computed gait velocity for first experiment

the entrance hall of the flat. The light barriers were mounted to the door frames in the living room and the bedroom. Doors to living room and bedroom were open, front door and bathroom door were closed. Before starting the experiment the scanner was calibrated with $k^{c}=100$ in the static environment according to the algorithm described in section IV-A. Results of this calibration are shown in figure 3 as black dots.

For the first part of the experiment participants were asked to walk directly from the living room to the bedroom and vice versa. For the second part, participants had to walk from the living room to the front door, lock the front door, and then walk to the bedroom. On their way back they were asked to reopen the front door before entering the living room. Participants were instructed to walk at their normal speed and to start from a standing position placing their first step right between the light barriers. In order to make results comparable while not restricting participants too much in their normal gait, the paths to walk included walking over a cross in the middle of the entrance hall. The first path had a length of $2.7 \mathrm{~m}$ measured by adding the distance from the middle of the living room's doorway to the mark and from their to the middle of bedroom's doorway. The second path was additional $2.2 \mathrm{~m}$ long. The time to walk the paths was recorded directly by the laser range scanner, by computing the time between reception of "on" states of the light barriers, and manually using a stopwatch. The manual measurement is thought to be the gold standard since it is commonly used in clinical environments e.g. during Timed Up \& Go.

\section{B. Results}

Results show that during the first part of the experiment (walking straightly from living room to bedroom and vice versa) self-selected gait velocity could be precisely computed from measurements of light barriers as well as from those of the laser range scanner compared to the stopwatch measurements. Gait velocity was computed by dividing the distance walked by the time measured. While the laser range scanners measured both values itself, distance had to be manually determined before the experiment for the other computations. Mean difference compared to the gold standard was only $0.023 \mathrm{~m} / \mathrm{s}$ for the light barriers and $0.063 \mathrm{~m} / \mathrm{s}$ for the laser range scanner. Standard deviations were $0.05 \mathrm{~m} / \mathrm{s}$ respectively $0.10 \mathrm{~m} / \mathrm{s}$. Figure 2 shows computed mean self-selected gait velocity for the first part of the experiment based on the time measured by the light barriers, the laser range scanner, and the stopwatch for each participant. Mean self-selected gait velocity across all participants was $0.99 \mathrm{~m} / \mathrm{s}$, ranging only from $0.90 \mathrm{~m} / \mathrm{s}$ for the first participant to $1.1 \mathrm{~m} / \mathrm{s}$ for the third participant.

The second part of the experiment was conducted in order to demonstrate the advantages of using a laser range scanner's measurements for computing self-selected gait velocity. Computations based on the time measured by the light barriers and the stopwatch for the second part were very imprecise due to the participants not walking directly from the living room to the bedroom but standing in between while opening or locking the front door. There is no possibility to compensate for this using only light barriers on room doors and in a real setup there would even be no chance to detect the longer walking distance. However, using the measurements of the laser range scanner, self-selected gait velocity could be computed precisely even for the second part of the experiment. This is due to the fact that the laser range scanner continuously measures the movement of the participants, measuring real distance walked and times walking and standing still. Figure 3 shows three measurement sets of the first participant recorded by the laser range scanner during the second part of the experiment. However, even while standing in front of the door the laser range scanner measures small movements e.g. because people move their feed slightly. Including these measurements into the gait velocity computation would heavily influence the results. Therefore, all measurements whose three consecutive movement vectors $\vec{d}_{k}, \vec{d}_{k+1}$, and $\vec{d}_{k+2}$ did not result in a distance walked longer than a certain threshold $d_{\text {thres }}$ were excluded from the gait velocity computation (equation 3 ). The threshold used for the experiments $d_{\text {thres }}=500 \mathrm{~mm}$ was determined empirically. By filtering measurements representing rest computed self-selected gait velocity for the second experiment had a mean error of only $0.01 \mathrm{~m} / \mathrm{s}$ and a standard deviation of $0.22 \mathrm{~m} / \mathrm{s}$ compared to the computation for the first experiment based on the laser range scanner's measurements.

$$
v_{k}=\left\{\begin{array}{l|l}
N a N \\
(2) & \mid \begin{array}{l}
\sum_{a=k}^{k+2} \vec{d}_{a} \mid<d_{\text {thres }} \\
\text { else }
\end{array}
\end{array}\right.
$$

\section{Discussion}

The conducted experiment was a first proof of concept for verifying that self-selected gait velocity may be reliably and precisely computed based on measurements of a laser range scanner in an unknown domestic environment. However, in order to sufficiently proof test-retest-reliability of the method, measurements with more and elderly participants over a 


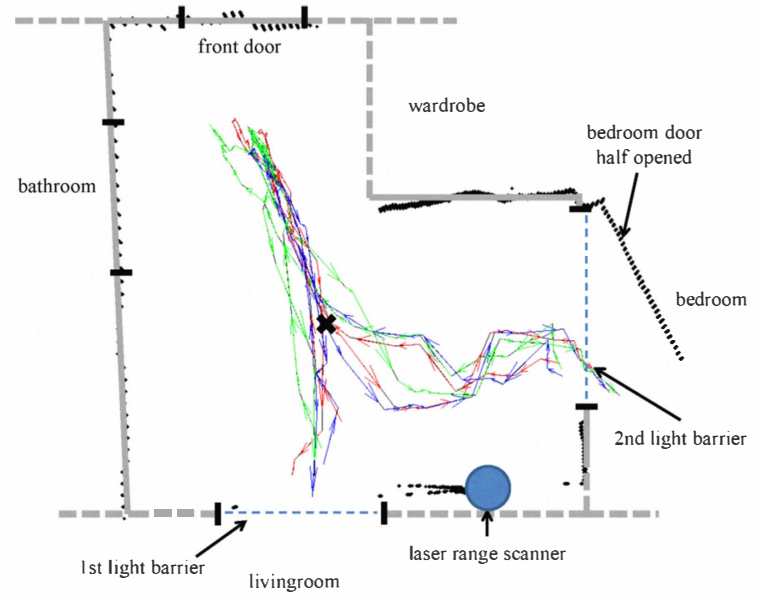

Fig. 3. Laser range scanner's measurements for three measurement sets of the second experiment displayed into the living lab's floor plan

longer period of time have to be evaluated.

The current version of the algorithm for computing gait velocity from laser range scanners' measurements has two serious limitations. First, the algorithm relies on a static environment during the calibration and measurement phases. If the environment is changed e.g. by opening a door after the initial calibration, the measurements are heavily effected. The algorithm is not capable of distinguishing more than one dynamic object at once. The second limitation regards the differences between the computed movement trajectory and the trajectory defined by the subjects center of mass mentioned in section IV. By incorporating background knowledge about the human respectively the human gait cycle, the computation may be further enhanced. Knowing that during the human gait cycle, especially during the gait phases mid stance and initial swing [44], one leg may be covered by the other may be used to guess missing measurement data and thus to compensate for the computed trajectory moving too far into the direction of the scanner.

Results of the experiment revealed possible new approaches to identifying residents, a common problem in ambient systems. Significant differences in computed self-selected gait velocity e.g. when comparing the first participant to all other participants were found. Thus, the gait velocity might be utilized for identification purposes. However, using only self-selected gait velocity may not work in all households especially if residents have similar age and health status. Another possibility for identification may be the resident's walking paths. The experiment revealed that participants chose characteristic walking paths (figure 3) which remained stable during measurement sets of each experiment part. However, by instructing participants to walk over a mark we may have influenced these results. Nevertheless, measurements of the laser range scanner are very precise and may be sufficient to compute more individual spatio-temporal parameters of gait which may be utilized for more reliable identification.
Self-selected gait velocity computed during the experiment was much slower than reference values reported in [16]. Butler et al. found an average gait velocity of $1.5 \mathrm{~m} / \mathrm{s}$ for healthy male people aged 20-39 years. However, the experiment set ups were very different. Values reported by Butler et al. may be regarded as capacity values since they were measured during a six meter walk in a clinical environment. Our experiment measured performance since it was conducted in a domestic environment and included walking around corners on a path with only $3 \mathrm{~m}$ length.

\section{CONCLuSion}

A novel approach to reliable and precise measurement of self-selected gait velocity in domestic environments has been presented. The approach is our first effort to developing an ambient assessment system capable of objectively measuring capacity and performance in mobility. The system is exclusively based on ambient sensors, therefore being totally unobtrusive and suitable even for demented people. The conducted experiment suggests that light barriers shall be used for measuring general trends in mobility covering a whole flat while the laser range scanner provides detailed measurements in a smaller area. The computation works without a priori knowledge of the environment and even if people walk around in a single room. Combining both approaches promises a good insight into the mobility of people. The theoretic concept was established and the algorithm for computing gait velocity from the scanner's measurement was described.

Currently, the algorithm still has serious limitations. Computation results may become more reliable by incorporating background knowledge about human gait. We are working on computing additional spatio-temporal parameters of gait by applying object identification techniques to the scanner's measurements. These parameters may be used for identification of residents which is future work as well. Further on, we are currently working on balance assessments utilizing the laser range scanner and additional sensors. Evaluation of the system with elderly people is already scheduled for the next year.

\section{ACKNOWLEDGMENT}

This work was partially funded by the German Ministry of Eduction and Research within the research project PAGE (grant \#01FCO8044).

\section{REFERENCES}

[1] T. M. Gill, C. S. Williams, and M. E. Tinetti, "Assessing risk for the onset of functional dependence among older adults: the role of physical performance." J Am Geriatr Soc, vol. 43, no. 6, pp. 603-609, Jun 1995.

[2] F. J. Imms and O. G. Edholm, "Studies of gait and mobility in the elderly." Age Ageing, vol. 10, no. 3, pp. 147-156, Aug 1981.

[3] B. G. Celler, T. Hesketh, W. Earnshaw, and E. Ilsar, "An instrumentation system for the remote monitoring of changes in functional health status of the elderly at home," in Proc. 16th Annual International Conference of the IEEE Engineering Advances: New Opportunities for Biomedical Engineers Engineering in Medicine and Biology Society, Nov. 3-6, 1994, pp. 908-909.

[4] E. Diczfalusy, "The demographic revolution and our common future." Maturitas, vol. 38, no. 1, pp. 5-14; discussion 14-5, Feb 2001. 
[5] M. Eisenmenger, O. Pötzsch, and B. Sommer. (2006 November) Bevölkerung deutschlands bis 2050 - 11. koordinierte bevölkerungsvorausberechnung. Statistisches Bundesamt Pressestelle, Wiesbaden. Statistisches Bundesamt 65180 Wiesbaden.

[6] L. Fratiglioni, D. D. Ronchi, and H. Agero-Torres, "Worldwide prevalence and incidence of dementia." Drugs Aging, vol. 15, no. 5, pp. 365-375, Nov 1999.

[7] A. D. International. (2009) World alzheimer report 2009. Online Http://www.alz.co.uk/research/files/World Alzheimer Report.pdf. [Online]. Available: http://www.alz.co.uk/research/files/World Alzheimer Report.pdf

[8] J. L. O'Loughlin, Y. Robitaille, J. F. Boivin, and S. Suissa, "Incidence of and risk factors for falls and injurious falls among the communitydwelling elderly." Am J Epidemiol, vol. 137, no. 3, pp. 342-354, Feb 1993.

[9] C. van Doorn, A. L. Gruber-Baldini, S. Zimmerman, J. R. Hebel, C. L. Port, M. Baumgarten, C. C. Quinn, G. Taler, C. May, J. Magaziner, and E. of Dementia in Nursing Homes Research Group, "Dementia as a risk factor for falls and fall injuries among nursing home residents." $J \mathrm{Am}$ Geriatr Soc, vol. 51, no. 9, pp. 1213-1218, Sep 2003.

[10] A. Wimo, B. Winblad, and L. Jönsson, "An estimate of the total worldwide societal costs of dementia in 2005." vol. 3, no. 2, April 2007, pp. 81-91. [Online]. Available: http://dx.doi.org/10.1016/j.jalz.2007.02.001

[11] L. M. Allan, C. G. Ballard, D. J. Burn, and R. A. Kenny, "Prevalence and severity of gait disorders in alzheimer's and non-alzheimer's dementias." J Am Geriatr Soc, vol. 53, no. 10, pp. 1681-1687, Oct 2005. [Online]. Available: http://dx.doi.org/10.1111/j.1532-5415.2005.53552.x

[12] J. Verghese, R. B. Lipton, C. B. Hall, G. Kuslansky, M. J. Katz, and H. Buschke, "Abnormality of gait as a predictor of non-alzheimer's dementia." N Engl J Med, vol. 347, no. 22, pp. 1761-1768, Nov 2002. [Online]. Available: http://dx.doi.org/10.1056/NEJMoa020441

[13] H. Stolze, S. Klebe, C. Baecker, C. Zechlin, L. Friege, S. Pohle, and G. Deuschl, "Prevalence of gait disorders in hospitalized neurological patients." Mov Disord, vol. 20, no. 1, pp. 89-94, Jan 2005. [Online]. Available: http://dx.doi.org/10.1002/mds.20266

[14] M. Montero-Odasso, M. Schapira, E. R. Soriano, M. Varela, R. Kaplan, L. A. Camera, and L. M. Mayorga, "Gait velocity as a single predictor of adverse events in healthy seniors aged 75 years and older." J Gerontol A Biol Sci Med Sci, vol. 60, no. 10, pp. 1304-1309, Oct 2005.

[15] C. O. Q. O. H. C. I. America and I. O. Medicine, Crossing the Quality Chasm: A New Health System for the 21st Century, 1st ed. National Academies Press, July 2001.

[16] A. A. Butler, J. C. Menant, A. C. Tiedemann, and S. R. Lord, "Age and gender differences in seven tests of functional mobility." J Neuroeng Rehabil, vol. 6, p. 31, 2009. [Online]. Available: http://dx.doi.org/10.1186/1743-0003-6-31

[17] O. Beauchet, G. Allali, G. Berrut, C. Hommet, V. Dubost, and F. Assal, "Gait analysis in demented subjects: Interests and perspectives." Neuropsychiatr Dis Treat, vol. 4, no. 1, pp. 155-160, Feb 2008.

[18] M. Beers and R. Berkow, Eds., The Merck Manual of Geriatrics. Merck \& Co., Inc., 2006. [Online]. Available: http://www.merck.com/mkgr/mmg/home.jsp

[19] D. Podsiadlo and S. Richardson, "The timed "up \& go": a test of basic functional mobility for frail elderly persons." J Am Geriatr Soc, vol. 39, no. 2, pp. 142-148, Feb 1991

[20] C. N. Scanaill, S. Carew, P. Barralon, N. Noury, D. Lyons, and G. M. Lyons, "A review of approaches to mobility telemonitoring of the elderly in their living environment." Ann Biomed Eng, vol. 34, no. 4, pp. 547-563, Apr 2006. [Online]. Available: http://dx.doi.org/10.1007/s10439-005-9068-2

[21] R. Williamson and B. J. Andrews, "Gait event detection for fes using accelerometers and supervised machine learning," IEEE Trans. Rehabil. Eng., vol. 8, no. 3, pp. 312-319, Sep. 2000.

[22] H. B. Menz, S. R. Lord, and R. C. Fitzpatrick, "Age-related differences in walking stability." Age Ageing, vol. 32, no. 2, pp. 137-142, Mar 2003.

[23] B. Auvinet, G. Berrut, C. Touzard, L. Moutel, N. Collet, D. Chaleil, and E. Barrey, "Reference data for normal subjects obtained with an accelerometric device." Gait Posture, vol. 16, no. 2, pp. 124-134, Oct 2002.

[24] W. Zijlstra, "Assessment of spatio-temporal parameters during unconstrained walking." Eur J Appl Physiol, vol. 92, no. 1-2, pp. 39-44, Jun 2004. [Online]. Available: http://dx.doi.org/10.1007/s00421004-1041-5
[25] H. Zhu, N. Maalej, J. G. Webster, W. J. Tompkins, P. Bach-Y-Rita, and J. J. Wertsch, "An umbilical data-acquisition system for measuring pressures between the foot and shoe," IEEE Trans. Biomed. Eng., vol. 37, no. 9, pp. 908-911, Sep. 1990.

[26] H. S. Zhu, J. J. Wertsch, G. F. Harris, J. D. Loftsgaarden, and M. B. Price, "Foot pressure distribution during walking and shuffling." Arch Phys Med Rehabil, vol. 72, no. 6, pp. 390-397, May 1991.

[27] J. M. Hausdorff, Z. Ladin, and J. Y. Wei, "Footswitch system for measurement of the temporal parameters of gait." J Biomech, vol. 28, no. 3, pp. 347-351, Mar 1995.

[28] M. M. Skelly and H. J. Chizeck, "Real-time gait event detection for paraplegic fes walking," IEEE Trans. Neural Syst. Rehabil. Eng., vol. 9, no. 1, pp. 59-68, Mar. 2001.

[29] I. P. I. Pappas, T. Keller, S. Mangold, M. R. Popovic, V. Dietz, and M. Morari, "A reliable gyroscope-based gait-phase detection sensor embedded in a shoe insole," IEEE Sensors J., vol. 4, no. 2, pp. 268-274, Apr. 2004.

[30] M. Hanlon and R. Anderson, "Real-time gait event detection using wearable sensors." Gait Posture, vol. 30, no. 4, pp. 523-527, Nov 2009. [Online]. Available: http://dx.doi.org/10.1016/j.gaitpost.2009.07.128

[31] S. Bamberg, A. Y. Benbasat, D. M. Scarborough, D. E. Krebs, and J. A. Paradiso, "Gait analysis using a shoe-integrated wireless sensor system," IEEE Trans. Inf. Technol. Biomed., vol. 12, no. 4, pp. 413-423, Jul. 2008.

[32] B. Huang, M. Chen, X. Shi, and Y. Xu, "Gait event detection with intelligent shoes," in Proc. International Conference on Information Acquisition ICIA '07, Jul. 8-11, 2007, pp. 579-584.

[33] M. Chen, B. Huang, and Y. Xu, "Intelligent shoes for abnormal gait detection," in Proc. IEEE International Conference on Robotics and Automation ICRA 2008, May 19-23, 2008, pp. 2019-2024.

[34] K. Kong and M. Tomizuka, "A gait monitoring system based on air pressure sensors embedded in a shoe," IEEE/ASME Trans. Mechat ronics, vol. 14, no. 3, pp. 358-370, Jun. 2009.

[35] M. Chan, C. Hariton, P. Ringeard, and E. Campo, "Smart house automation system for the elderly and the disabled," in Proc. IEEE International Conference on Systems, Man and Cybernetics Intelligent Systems for the 21st Century, vol. 2, Oct. 22-25, 1995, pp. 1586-1589.

[36] K. Cameron, K. Hughes, and K. Doughty, "Reducing fall incidence in community elders by telecare using predictive systems," in Proc. 19th Annual International Conference of the IEEE Engineering in Medicine and Biology Society, vol. 3, Oct. 30-Nov. 2, 1997, pp. 1036-1039.

[37] M. Pavel, T. Hayes, I. Tsay, D. Erdogmus, A. Paul, N. Larimer, H. Jimison, and J. Nutt, "Continuous assessment of gait velocity in parkinson's disease from unobtrusive measurements," in Proc. 3rd International IEEE/EMBS Conference on Neural Engineering CNE '07, May 2-5, 2007, pp. 700-703.

[38] P. Srinivasan, D. Birchfield, G. Qian, and A. Kidané, "A pressure sensing floor for interactive media applications," in ACE '05: Proceedings of the 2005 ACM SIGCHI International Conference on Advances in computer entertainment technology. New York, NY, USA: ACM, 2005, pp. 278281.

[39] L. Middleton, A. A. Buss, A. Bazin, and M. S. Nixon, "A floor sensor system for gait recognition," in Proc. Fourth IEEE Workshop on Automatic Identification Advanced Technologies, Oct. 17-18, 2005, pp. 171-176.

[40] J. Tung, W. Gage, K. Zabjek, D. Brooks, D. Maki, A. Mihailidis, G. R. Fernie, and W. E. Mcllroy, "iwalker: A 'realworld' mobility assessment tool," in 30th Canadian Medical \& Biological Engineering Society, 2007. [Online]. Available: http://www.ot.utoronto.ca/iatsl/projects/iwalker.htm.

[41] P. W. Kong and H. D. Heer, "Wearing the f-scan mobile in-shoe pressure measurement system alters gait characteristics during running." Gait Posture, vol. 29, no. 1, pp. 143-145, Jan 2009. [Online]. Available: http://dx.doi.org/10.1016/j.gaitpost.2008.05.018

[42] R. J. Orr and G. D. Abowd, "The smart floor: a mechanism for natural user identification and tracking," in $C H I$ ' $O 0$ : $C H I$ 'OO extended abstracts on Human factors in computing systems. New York, NY, USA: ACM, 2000, pp. 275-276.

[43] G. Virone, D. Istrate, M. Vacher, N. Noury, J. F. Serignat, and J. Demongeot, "First steps in data fusion between a multichannel audio acquisition and an information system for home healthcare," in In IEEE Conference on Engineering in Medecine and Biology Society, 2003, pp. 1364-1367.

[44] J. Perry, Gait Analysis: Normal and Pathological Function. Slack, Inc., 1992. 